\title{
AN INFINITE DIMENSIONAL VERSION OF A THEOREM OF BERNSTEIN
}

\author{
GUILLERMO RESTREPO
}

1. Introduction. Let $P\left(R^{n}\right)$ be the algebra of polynomials in $n$ variables with the topology of uniform convergence on bounded sets of a function $f$ and its derivative $f^{\prime}$. A classical theorem of Bernstein says that the closure of $\mathcal{P}\left(R^{n}\right)$ is the algebra $\mathfrak{e}^{1}\left(R^{n}\right)$ of all real-valued functions of class $C^{1}$. In other words, for every $f \in \mathbb{C}^{1}\left(R^{n}\right)$ there is a sequence $\left\{p_{n}\right\}$ of polynomials such that $p_{n} \rightarrow f$ uniformly on bounded sets and $f_{n}^{\prime} \rightarrow f^{\prime}$ uniformly on bounded sets. In this paper we define the algebra $\rho(X)$ of polynomials in a Banach space $X$ and determine its closure for a restricted class of reflexive Banach spaces (Theorem 8). Thus, Theorem 8 answers a question raised by the author in [2]. In what follows, weak convergence in $X$ will be denoted by $x_{n} \rightarrow x$ and strong convergence by $x_{n} \rightarrow x$. Henceforth we will assume all Banach spaces to be separable.

2. Polynomials in Banach spaces. Let $X$ be a Banach space and let $X^{*}$ be its dual. If $u=\left(u_{1}, \cdots, u_{n}\right) \in\left(X^{*}\right)^{n}$ and $\alpha=\left(\alpha_{1}, \cdots, \alpha_{n}\right)$ is a sequence of positive integers, we define $u^{\alpha}$ to be the real valued function $u^{\alpha}(x)=\left(u_{1}(x)\right)^{\alpha_{1}} \cdots\left(u_{n}(x)\right)^{\alpha_{n}}$ and $|\alpha|=\alpha_{1}+\cdots+\alpha_{n} . A$ polynomial in $X$ is a function $p: X \rightarrow R$ of the form $p=\sum_{|\alpha|_{s m}} a_{\alpha} u^{\alpha}$ where $a_{\alpha}$ is a real number.

Let $X, Y$ be Banach spaces. An operator $T: X \rightarrow Y$ is weakly continuous if $T$ is continuous from the weak topology in $X$ to the norm topology in $Y$.

Theorem 1. Let $X$ be a Banach space and let $p$ be a polynomial. Then

(i) $p$ is weakly continuous.

(ii) The derivative $p^{\prime}: X \rightarrow X^{*}$ is weakly continuous.

(iii) $p^{\prime}(X)$ is finite dimensional.

Proof. (i) follows from the definition of $p$. In order to prove (ii) and (iii) we simply calculate $p^{\prime}(x)$. If $p(x)=u(x) v(x)$, where $u, v \in X^{*}$, then $p^{\prime}(x)=u(x) v+v(x) u$, and if $p(x)=(u(x))^{k}$ then $p^{\prime}(x)=k(u(x))^{k-1} u$. Now, by induction, one can show if $p=u^{\alpha}=u_{1}^{\alpha_{1}} \cdots u_{n}^{\alpha_{n}}$ then $p^{\prime}(x)$ is of the form $\sum_{i=1}^{n} \lambda_{i}(x) u_{i}$ where $\lambda_{i}$ is a weakly continuous real valued function. In this case $p^{\prime}$ satisfies (ii) and (iii). The general case follows from the previous ones.

Received by the editors September 23, 1968 and, in revised form, March 10, 1969. 
TheORem 2. Let $X, Y$ be Banach spaces and $f: X \rightarrow Y$ continuous. Let $P: X \rightarrow Y$ be linear and bounded and such that $P(X)$ is finite dimensional. Then

(i) $f \circ P$ is weakly continuous.

(ii) $(f \circ P)^{\prime}=P^{*} \circ f^{\prime} \circ P$.

Proof. (i) Let $x_{n} \rightarrow x\left(x_{n}\right.$ converges weakly to $\left.x\right)$. Then $P x_{n} \rightarrow P x$ since $P$ is linear. Furthermore, $P x_{n} \rightarrow P x$ since $P(X)$ is finite dimensional, so $f P\left(x_{n}\right) \rightarrow f P(x)$ since $f$ is continuous.

(ii) A trivial calculation.

Theorem 3. Let $X$ be a reflexive Banach space. Then $f: X \rightarrow R$ is weakly continuous on bounded sets if and only there is a sequence $\left\{p_{n}\right\}$ of polynomials such $p_{n} \rightarrow f$ uniformly on bounded sets.

Proof. Every set of the form $\{x \mid\|x\| \leqq M\}$ is weakly compact, so $f$ is weakly continuous if $p_{n} \rightarrow f$ uniformly on bounded sets. Let us prove the second part. The theorem of Stone-Weierstrass can be used to show that for every continuous function $f$ on bounded sets and every integer $n$ there is a polynomial $p_{n}$ such that $\left|f(x)-p_{n}(x)\right|<1 / n$ for all $x$ such that $\|x\| \leqq n$. The sequence $\left\{p_{n}\right\}$ converges to $f$ uniformly on bounded sets. This concludes the proof.

REMARK. Weakly continuous on bounded sets means: the restriction of $f$ to any bounded subset of $X$ is relatively weakly continuous. If a function is weakly continuous, then it is weakly continuous on bounded sets. The converse is not true. Let $X=H$ be a Hilbert space, let $e_{1}, e_{2}, \cdots, e_{n}, \cdots$ be a basis of $H$ and let

$$
f(x)=\sum_{n=1}^{\infty} \frac{\left(e_{n}(x)\right)^{n}}{(2 n) !} .
$$

If this function were weakly continuous at the origin of $H$ then it would be bounded on some subspace $M$ of finite codimension in $H$. For $x \in M$ fixed,

$$
g(z)=\sum_{n=1}^{\infty} \frac{\left(e_{n}(x)\right)^{n}}{(2 n) !} z^{n}
$$

is an entire function of order $1 / 2$ in $z$. Such a function cannot be bounded for all real $z$ unless it is a constant, in this case zero. Hence $M=\{0\}$ which is a contradiction. I am grateful to the referee for pointing out this nice example and for other constructive comments.

We say that a Banach space $X$ has property (B) if there is a sequence $P_{n}: X \rightarrow X$ of bounded linear operators such that 
(i) $P_{n}(X)$ is finite dimensional for each $n$.

(ii) $P_{n}^{2}=P_{n}$.

(iii) $P_{n}(x) \rightarrow x$ for every $x \in X$.

(iv) $P_{n}^{*}(u) \rightarrow u$ for every $u \in X^{*}$.

Every Banach space with a biorthogonal basis has property (B). In particular, every Hilbert space has property (B). The theorem below has been proved by Vainberg in [1] for reflexive Banach spaces with a biorthogonal basis. Our proof is similar to his.

THEOREM 4. Let $X$ be a reflexive Banach space with property (B). Let $T: X \rightarrow X^{*}$ be a continuous operator. Then $T$ is weakly continuous on bounded sets if and only if $\mathrm{T} \circ P_{n} \rightarrow T$ uniformly on bounded sets.

Proof. By the uniform boundness principle there is a constant $M$ such that $\left\|P_{n}\right\| \leqq M$ and $\left\|P_{n}^{*}\right\| \leqq M$ for all $n$. Let us show that if $x_{n} \rightarrow x$ then $P_{n} x_{n} \rightarrow x$. Now, $\left\langle P_{n} x_{n}, u\right\rangle=\left\langle x_{n}, P_{n}^{*} u\right\rangle=\left\langle x_{n}, u\right\rangle+\left\langle x_{n}, P_{n}^{*} u-u\right\rangle$. Since $P_{n}^{*} u \rightarrow u$, and $\left\{x_{n}\right\}$ is bounded, it follows that $\left\langle P_{n} x_{n}, u\right\rangle \rightarrow 0$ for every $u \in X^{*}$.

Let us assume that $T$ is weakly continuous on bounded sets and suppose that $T \circ P_{n}$ does not converge to $T$ uniformly on bounded sets. Then, for some $\epsilon>0$ and for every integer $k$ there exist an integer $n_{k}$ and a point $x_{k}$ such that $\left\|x_{k}\right\| \leqq M$ for some $M>0$ and $\left\|T P_{n_{k}} x_{k}-T x_{k}\right\|$ $\geqq \epsilon, k=1,2, \cdots$. Since $X$ is reflexive, there is a subsequence (still denoted by $\left\{x_{k}\right\}$ ) such that $x_{k} \rightarrow x_{0}$ for some $x_{0}$. This would imply that $P_{n_{k}} x_{k} \rightarrow x_{0}$ and $T P_{n_{k}} x_{k} \rightarrow T x_{0}$. Thus, we have reached a contradiction. Let us assume that $T \circ P_{n} \rightarrow T$ uniformly on bounded sets. Since $T \circ P_{n}$ is weakly continuous by Theorem 2 and $\{x \mid\|x\| \leqq M\}$ is weakly compact for every $M>0$, it follows that $T$ is weakly continuous on bounded sets.

3. Uniformly differentiable functions. Let $X$ and $Y$ be Banach spaces and let $f: X \rightarrow Y$ be of class $C^{1}$. We say that $f$ is uniformly differentiable in a subset $A \subset X$ if for every $\epsilon>0$ there is some $\delta>0$ such that

$$
\left\|f(a+h)-f(a)-\left\langle f^{\prime}(a), h\right\rangle\right\| \leqq \epsilon\|h\|
$$

whenever $\|h\| \leqq \delta$ for all $a \in A$.

Theorem 5. Let $X$ be a reflexive Banach space with property (B). Let $f: X \rightarrow R$ be weakly continuous and uniformly differentiable on bounded sets. Let $T=f^{\prime}: X \rightarrow X^{*}$. Then $P_{n}^{*} \circ T \rightarrow T$ uniformly on bounded sets.

Proof. Let $I: X \rightarrow X$ be the identity operator and let $h=\left(I-P_{n}\right)(z)$. 
Let $O(x, h)=f(x+h)-f(x)-\langle T(x), h\rangle$. Since $\langle T x, h\rangle=\left\langle\left(I-P_{n}^{*}\right) T x, h\right\rangle$ we get,

$$
\begin{aligned}
\left|\left\langle\left(I-P_{n}^{*}\right) T x, h\right\rangle\right| \leqq & |f(x+h)-f(x)|+|O(x, h)| \\
\leqq & \left|f(x+h)-f\left(P_{n}(x+h)\right)\right| \\
& +\mid f\left(P_{n}(x+h)-f(x)|+| O(x, h) \mid .\right.
\end{aligned}
$$

Let $\epsilon>0$ be given and let $M>0$. Since $f$ is uniformly differentiable on bounded sets there is some $\delta>0$ such that

$$
|O(x, h)|<(\epsilon / 3)\|h\| \quad \text { if }\|h\|<\delta
$$

for all $x$ such that $\|x\| \leqq M$. Now, by the uniform boundness principle there is some $N$ such that $\left\|I-P_{n}\right\| \leqq N$ for all $n$. Since $f$ is weakly continuous, there is some $n_{0}$ (see Theorem 4) such that

$$
\begin{aligned}
& \left|f(x+h)-f\left(P_{n}(x+h)\right)\right|<\epsilon \delta / 3 N \\
& \left|f(x)-f\left(P_{n} x\right)\right|<\epsilon \delta / 3 N \\
& n \geqq n_{0}
\end{aligned}
$$

for all $x$ such that $\|x\| \leqq N$ and all $h$ such that $\|h\|<\delta$. Let $y \in X$ such that $\|y\|=1$ and let $h=(\delta / N)\left(I-P_{n}\right) y$. then $\|h\| \leqq \delta$ and

$$
\begin{aligned}
\left|\left\langle\left(I-P_{n}^{*}\right) T x, y\right\rangle\right| & =\left|\left\langle\left(I-P_{n}^{*}\right) T x,\left(I-P_{n}\right) y\right\rangle\right| \\
& =(M / \delta)\left|\left\langle\left(I-P_{n}^{*}\right) T x, h\right\rangle\right| ;
\end{aligned}
$$

from (1), (2), (3) and (4) we get

$$
\left\|\left(I-P_{n}^{*}\right) T x\right\|<\epsilon \quad n \geqq n_{0}
$$

for all $x$ such that $\|x\| \leqq M$. This concludes the proof.

Theorem 6. Let $X$ be a reflexive Banach. Assume $f: X \rightarrow R$ is weakly continuous and uniformly differentiable on bounded sets. Then $f^{\prime}$ $=T: X \rightarrow X^{*}$ is weakly continuous.

Proof. We have to show that $x_{n} \rightarrow x$ implies $T x_{n} \rightarrow T x$. Since $\left\{x_{n}\right\}$ is bounded, we can assume that everything takes place in some bounded set $B=\{x \mid\|x\| \leqq M\}$. Let $\epsilon>0$. By uniform differentiability we can make

$$
\|O(x, h)\|<4^{-1} \epsilon\|h\|
$$

if $\|h\| \leqq \delta<1$ for some $\delta$ and all $x \in B$. Since $f$ is weakly uniformly continuous in $2 \mathrm{~B}$, there is a weak neighborhood $V(0)$ such that,

$$
|f(y)-f(z)|<4^{-1} \delta \epsilon
$$

for all $y, z \in 2 B$ such that $y-z \in V(0)$. Now, 


$$
\begin{aligned}
\left\langle T x_{n}, h\right\rangle & =f\left(x_{n}+h\right)-f\left(x_{n}\right)-O\left(x_{n}, h\right) \\
\langle T x, h\rangle & =f(x+h)-f(x)-O(x, h)
\end{aligned}
$$

and

$$
\begin{aligned}
\left|\left\langle T x_{n}-T x, h\right\rangle\right| \leqq & \left|f\left(x_{n}+h\right)-f(x+h)\right|+\left|f\left(x_{n}\right)-f(x)\right| \\
& +\left|O\left(x_{n}, h\right)\right|+|O(x, h)| .
\end{aligned}
$$

Let $n_{0}$ be such that $x_{n}-x_{0} \in V$ if $n \geqq n_{0}$. From (1), (2) and (3) we obtain

$$
\left|\left\langle T x_{n}-T x, h\right\rangle\right|<\epsilon \delta
$$

if $n \geqq n_{0}$ and $\|h\|=\delta$. This shows that $\left|\left\langle T x_{n}-T x, h\right\rangle\right|<\epsilon$ if $n \geqq n_{0}$ and $\|h\|=1$. Therefore, $\left\|T x_{n}-T x\right\|<\epsilon$ if $n \geqq n_{0}$. This concludes the proof.

Theorem 7. Let $X$ be a reflexive Banach with property (B). Let $f: X \rightarrow R$ be weakly continuous and uniformly differentiable on bounded sets. Then $P_{n}^{*} T P_{n} \rightarrow T$ uniformly on bounded sets.

Proof. We have for any $x \in X$

$$
\left\|T x-P_{n}^{*} T P_{n}(x)\right\| \leqq\left\|T x-P_{n}^{*} T x\right\|+\left\|P_{n}^{*} T x-P_{n}^{*} T P_{n} x\right\| .
$$

The first term in the right-hand side converges to zero uniformly on bounded sets (Theorem 5). The second term is lesser or equal than $\left\|P_{n}^{*}\right\|\left\|T x-T P_{n} x\right\|$ which converges to zero uniformly on bounded sets by Theorems 6 and 4 and the fact that $\left\|P_{n}\right\| \leqq M$ for some constant $M$ and all $n$. This concludes the proof.

\section{The main theorem.}

THEOREM 8. Let $X$ be a reflexive Banach space with property (B). Let $\odot(X)$ be the algebra of polynomials in $X$ with the topology of uniform convergence on bounded sets of a function and its derivative. Then the closure of $(X)$ is the algebra of weakly continuous functions on bounded sets which are uniformly differentiable on bounded sets.

Proof. Let $f$ be weakly continuous and uniformly differentiable on bounded sets, and let $f_{n}=f \circ P_{n}$. Then $f_{n}^{\prime}=P_{n}^{*} \circ f^{\prime} \circ P_{n}$ (Theorem 2), $f_{n} \rightarrow f$ uniformly on bounded sets (Theorem 4) and $f_{n}^{\prime} \rightarrow f^{\prime}$ uniformly on bounded sets (Theorem 7). Therefore, we can assume that that $f=g \circ P$ where $P$ is a projection into some finite-dimensional subspace and $g$ is uniformly differentiable on bounded sets. Let $P(X)=H$ and let $h$ be the restriction of $g$ to $H$, that is, $h(x)=g(x)$ for all $x \in H$. Since $H$ is finite dimensional there is a sequence $\left\{q_{n}\right\}$ of 
polynomials in $H$ such that $q_{n} \rightarrow h, q_{n}^{\prime} \rightarrow h^{\prime}$ uniformly on bounded sets (this follows from a classical theorem of Bernstein). Let $p_{n}=q_{n} \circ P$. Then,

$$
\left|f(x)-p_{n}(x)\right|=\left|g P(x)-q_{n} P(x)\right| \rightarrow 0
$$

uniformly on bounded sets. Now, let $u \in X$ such that $\|u\|=1$. Then,

$$
\begin{aligned}
\left|\left\langle f^{\prime}(x)-p_{n}^{\prime}(x), u\right\rangle\right| & =\left|\left\langle g^{\prime} P x-q_{n}^{\prime} P x, P u\right\rangle\right| \\
& =\left|\left\langle h^{\prime} P(x)-q_{n}^{\prime} P x, P u\right\rangle\right| \\
& \leqq\|P\|\left\|h^{\prime} P(x)-q_{n}^{\prime} P(x)\right\| .
\end{aligned}
$$

Therefore, $\left\|f^{\prime},(x)-P_{n}^{\prime}(x)\right\| \leqq\|P\|\left\|h^{\prime} P(x)-q_{n}^{\prime} P(x)\right\| \rightarrow 0$ uniformly on bounded sets. This concludes the proof.

\section{REFERENCES}

1. M. M. Vainberg, Variational methods for the study of nonlinear operators, HoldenDay, San Francisco, Calif., 1964.

2. G. Restrepo, Remarks on weakly continuous functions in Banach spaces, Rev. Colombiana Mat. (to appear).

University of Puerto Rico 